\title{
Reflexive attention attenuates change blindness (but only briefly)
}

\author{
D. T. SMITH AND T. SCHENK \\ University of Durham, Stockton-on-Tees, England
}

\begin{abstract}
Humans are remarkably insensitive to large changes in a visual display if the change occurs simultaneously with a secondary perceptual event. A widely held view is that this change blindness occurs because the secondary perceptual event prevents the change from capturing attention. However, whereas some studies have shown that top-down attentional priming can attenuate change blindness, the evidence regarding the effect of bottomup attentional capture on change blindness is less clear-cut. Here, we compare the effects of attentional capture on change detection with participants' performance on a well-established attentional paradigm (a Posner-style cuing task). Experiment 1 established the time course of attentional capture in our paradigm. Experiment 2 demonstrated that this attentional capture was associated with facilitated change detection at short (150-msec), but not long (480-msec), latencies. These data show that reflexive attentional shifts facilitate change detection and are consistent with the view that shifts of attention are a necessary precondition for visual awareness.
\end{abstract}

We are constantly receiving sensory input from our eyes, ears, and skin, yet only a small portion of this sensory information ever arrives in our conscious awareness. The question of what determines access to conscious awareness has been the subject of research interest for more than a century yet remains one of the most controversial issues in psychology. This controversy has, in part, arisen because it is so difficult to disentangle awareness from other cognitive processes, making it very difficult to study in isolation. However, in recent times, a paradigm has been developed that appears to offer a new and effective way of studying visual awareness - principally, by investigating situations in which participants fail to become aware of sudden and obvious changes in the world.

Under normal circumstances changes to our environment are easy to spot (a bird flying from its perch, for example). However, under certain circumstances, even large changes can go unnoticed. Specifically, observers frequently fail to become aware of a change when this change occurs simultaneously with another perceptual event. These perceptual events can include an eye movement (saccade; Bridgeman, Hendry, \& Stark, 1975; McConkie \& Zola, 1979), eye blinks (O'Regan, Deubel, Clark, \& Rensink, 2000), the occlusion of a visual display with a mask (Pashler, 1988; Phillips, 1974; Rensink, O'Regan, \& Clark, 1997), or even a sudden onset that attracts attention but does not occlude the location of the change (O'Regan, Rensink, \& Clark, 1999). This phenomenon of change blindness has generated considerable interest and has become the focus of much recent research, since it appears to offer researchers the opportunity to investigate the precise conditions required for visual events, such as changes, to enter awareness.
One specific claim that has emerged from the study of change blindness is that in order for a visual stimulus to enter into awareness, it must first be attended (e.g., Simons \& Rensink, 2005). In other words, attention is a necessary precondition for visual awareness. This conclusion is based principally on the observation that changes to interesting items are more easily detected than changes to less interesting items (Kelley, Chun, \& Chua, 2003; Rensink et al., 1997) and that cuing the location of a change by informing the observer of the location of the change improves change detection (Rensink et al., 1997). This bold conclusion is consistent with the idea that attention acts to select certain sensory information for higher processing while inhibiting the processing of irrelevant sensory information.

However, it is worth exercising some caution before concluding, on the basis of these studies, that attention is required for visual awareness. It is less than ideal to use explicit cues to manipulate attention in order to examine the link between attention and awareness. Explicit cues guide attention by making the observer aware of the importance of a specific part of the scene. It is, therefore, not entirely surprising that calling part of a scene into visual awareness also makes subsequent changes to that part of the scene more likely to enter into visual awareness. A more convincing way to examine the link between attention and awareness is to use an implicit or exogenous attentional manipulation that provides no information about the relative importance of specific parts of a scene. A typical example of an exogenous manipulation of attention is the Posner cuing task. Here, the sudden onset of a peripheral stimulus (cue) triggers a reflexive shift of attention to the location of the cue, producing faster and more efficient processing

D. T. Smith, daniel.smith2@durham.ac.uk 
of visual information at this location. These reflexive shifts of attention follow a well-established time course: The cue produces a short-lived facilitatory effect that peaks at $\sim 150$ msec (Müller \& Rabbitt, 1989) and is superseded by a more sustained inhibitory effect after $\sim 300 \mathrm{msec}$ (e.g., Maylor \& Hockey, 1985; Posner \& Cohen, 1984).

Scholl (2000) employed just such a reflexive paradigm to study how change blindness is affected by attention and observed enhanced change detection at the location of a peripheral cue (which took the form of a sudden onset). Although Scholl's data appear to support the notion that reflexive attention facilitates change detection, there is reason to be cautious in drawing this conclusion. Specifically, Scholl reported a sustained facilitatory effect that was first observed $480 \mathrm{msec}$ after the cue onset and persisted for several seconds. In other words, Scholl observed a facilitatory effect after reflexive attention had been withdrawn from the cued location. Thus, it is impossible to attribute the effects observed by Scholl to the facilitatory effects of a reflexive attention shift. In this context, it is important to note that Scholl did not record or control participants' eye movements. It is, therefore, quite possible that the participants in his experiment moved their eyes to the location of the cue. A change presented at the location of the cue would then fall directly into their fovea. This could easily explain why change detection was significantly increased at the location of the cue and also why this effect lasted for several seconds. Given the well-established association between eye movements and covert attention (e.g., Deubel \& Schneider, 1996; Hoffman \& Subramaniam, 1995; Shepherd, Findlay, \& Hockey, 1986), the confounding of eye position with locus of attention presents a major problem for the interpretation of effects, since it is impossible to rule out the possibility that the improved rates of change detection at the attended location are simply the consequence of improved vision at the center of vision. The failure to control for eye movements is further compounded by the use of very long presentation times that actually facilitate the use of overt searching strategies. For example, trials in Scholl's experiment often lasted for several seconds. In this case, participants can easily move around their eyes. Moreover, unless the stimulus presentation is temporally constrained, it becomes impossible to compare the time course of change detection facilitation with the time course of attentional facilitation known from the Posner (Posner \& Cohen, 1984) cuing task.

In order to address these issues and convincingly demonstrate that attention per se is required to become aware of a change, it is necessary to employ a change blindness task that directs attention in a nonpredictive fashion, which is temporally constrained and dissociates covert attention from overt eye movements. One task that meets these requirements is the Posner cuing task, in which a peripheral, nonpredictive spatial cue captures attention, leading to faster detection of subsequently presented targets (Posner \& Cohen, 1984). This task is ideal, since the cue does not predict the location of the target and, therefore, does not manipulate expectancies in an implicit or endogenous fashion. Furthermore, the paradigm has been intensively studied and has a well-established time course. Finally, the task requires that fixation be maintained in the center of the display and that eye movements be forbidden, so that they cannot be confounded with covert attention.

A clear prediction can be drawn from the biphasic effects observed in the Posner (Posner \& Cohen, 1984) paradigm. If attention is necessary for awareness of change, change detection should be easier at the location of a peripheral precue, but only during the $150 \mathrm{msec}$ when attention is reflexively deployed to the cued location. At longer latencies, when attention is withdrawn from the cued location, change detection should return to baseline levels (or potentially get worse, since inhibition of return effects bias attention away from the cued location). Two experiments were conducted to test this prediction. Experiment 1 established the time course of attentional allocation in response to a peripheral precue. Experiment 2 then measured the impact of this attentional shift on change detection. Eye movements were recorded in both experiments to ensure that the locus of attention was never confounded with gaze direction.

\section{EXPERIMENT 1}

The aim of Experiment 1 was to establish the time course of the reflexive attentional shifts elicited during the sudden-onset paradigm. In order to make this task as similar as possible to the change blindness task, the display was occluded for $80 \mathrm{msec}$ prior to target onset. It was predicted that the sudden onset would produce a short-lived perceptual facilitation at the cued location that should be observable at a cue-target stimulus onset asynchrony (SOA) of 150 msec (Müller \& Rabbitt, 1989). At the longer 480-msec cue-target SOA, the facilitatory effect should be masked by inhibitory processes (Posner \& Cohen, 1984). Note that the $480-\mathrm{msec}$ SOA was the same as that employed by Scholl (2000).

\section{Method}

Participants. Six participants were recruited from the University of Durham ( 3 of them female). Their ages ranged from 23 to 42 years (mean age, 29 years). All the participants had normal or correctedto-normal vision.

Apparatus. The stimuli were generated using a VSG 2/5 graphics card (CRS, Rochester, England) and were displayed on a Sony Trinitron monitor with a $100-\mathrm{Hz}$ refresh rate. Eye movements were recorded using a Cambridge Research Systems Video Eyetracker Toolbox (2.1) sampling at $50 \mathrm{~Hz}$. Participant responses were recorded using a standard keyboard.

Stimuli. The array of objects consisted of a black fixation point and six white rectangular boxes $\left(1.14^{\circ} \times 0.57^{\circ}\right)$ on a gray background. The boxes could appear at eccentricities of $11.4^{\circ}, 8.5^{\circ}$, or $5.7^{\circ}$ from fixation along the $x$-axis and $8.5^{\circ}, 6.7^{\circ}$, or $4.8^{\circ}$ along the $y$-axis (note that this means that no array item could appear within $5.7^{\circ}$ of fixation). There were two constraints on the position of the boxes. First, there could be no two boxes at the same eccentricity on the $x$-axis. Second, there should be three boxes on the left and three boxes on the right of fixation. Boxes were randomly oriented horizontally or vertically. The probe stimulus consisted of a black asterisk $\left(0.3^{\circ} \times 0.2^{\circ}\right)$.

Procedure. The participants were presented with a display consisting of a gray background with a black fixation point at the center. After 1,500 msec, five white rectangles appeared, followed $500 \mathrm{msec}$ later by a sixth rectangle. After a variable latency ( 70 or $400 \mathrm{msec}$ ), 


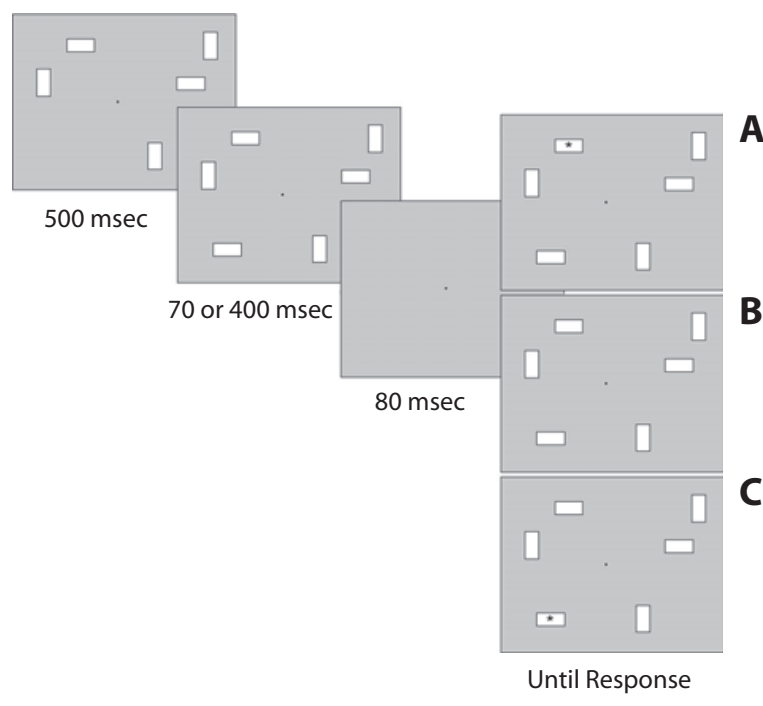

Figure 1. Procedure during Posner cuing paradigm (Experiment 1). On all trials, we first presented an array of rectangular objects for 500 msec. Then a new rectangular object was presented for $\mathbf{7 0}$ or $\mathbf{4 0 0} \mathbf{m s e c}$ (in this case, it is the new horizontally oriented rectangle in the lower left corner). Afterward, a blank mask was presented. The probe was presented during the final display, which lasted until the observer gave the response. These displays are illustrated in panels $\mathrm{A}-\mathrm{C}$ for the different trial types. Panel A shows invalid trials (target appeared at an uncued location), panel B shows no-target trials, and panel $C$ shows valid trials (target appeared at the cued location-i.e., in the new object in the lower left corner). The target remained visible until response.

the display was occluded for 80 msec by a gray mask, after which the display of six rectangles reappeared. On $66 \%$ of the trials, a black asterisk appeared in one of the rectangles (probe trials); on the remaining $34 \%$ of the trials, no probe appeared (catch trials). On 16\% of the probe trials, the asterisk appeared at the location of the sudden onset (valid trials). On the remaining $84 \%$ of the probe trials, the asterisk occurred at one of the uncued locations (invalid trials). This display remained present until the participants made a response. The participants were told to report whether or not they saw the probe by pressing the appropriate response key as quickly as possible. Each participant completed 10 practice trials and 320 experimental trials ( 80 catch trials, 40 valid trials, and 200 invalid trials). Figure 1 illustrates the course of events on a typical trial.

\section{Results}

Data analysis. Responses were filtered to remove any trials on which the participants made a saccade before the onset of the mask and responses that were more than 2.5 standard deviations longer than an individual's mean reaction time (RT). Any eye movement with an amplitude of $>2^{\circ}$ was considered to be a saccade. These procedures resulted in the rejection of $2.6 \%$ of the trials $(0.9 \%$ RT error, $1.7 \%$ saccades). For the analysis of RTs, the data were further filtered to remove incorrect responses, resulting in the rejection of a further $6.6 \%$ of the trials.

Accuracy. The participants mean accuracy scores were subjected to a 2 (SOA: 150 or $480 \mathrm{msec}$ ) $\times 2$ (validity: valid or invalid) repeated measures ANOVA. This analysis revealed no significant main effects and no significant interactions.
Reaction time. The participants' mean RTs on the probe trials were subjected to a 2 (SOA: 150 or $480 \mathrm{msec}) \times 2$ (validity: valid or invalid) repeated measures ANOVA. This analysis revealed a significant main effect of validity $[F(1,5)=7.75, p<.05]$ and a significant validity $\times$ SOA interaction $[F(1,5)=8.14, p<.05]$. Planned contrasts (paired sample $t$ tests) revealed that interaction was driven by a significant cuing effect at the $150-\mathrm{msec}$ latency, so that responses at the cued location were faster than those at the uncued location [406 vs. $434 \mathrm{msec} ; t(5)=2.89, p<$ $.05]$. This cuing effect was not present at the $480-\mathrm{msec}$ SOA [411 vs. $408 \mathrm{msec} ; t(5)=0.943, p=.38$ ]. Figure 2 illustrates this interaction.

\section{Discussion}

The results of Experiment 1 demonstrate that perceptual performance was facilitated at the location of the sudden onset, relative to the other locations, but only at short $(150-\mathrm{msec})$ latencies. In contrast, there was no significant cuing effect at the longer $480-\mathrm{msec}$ latency. These data demonstrate that the sudden onset produced a reflexive shift of attention but that the cuing effect caused by this attentional shift was not sustained. Since there was no baseline condition, it is impossible to determine whether the cuing effect reflects improved performance at the cued location or inhibited performance at the uncued locations. Indeed, it is likely that the cuing effect was the product of some combination of these pro-

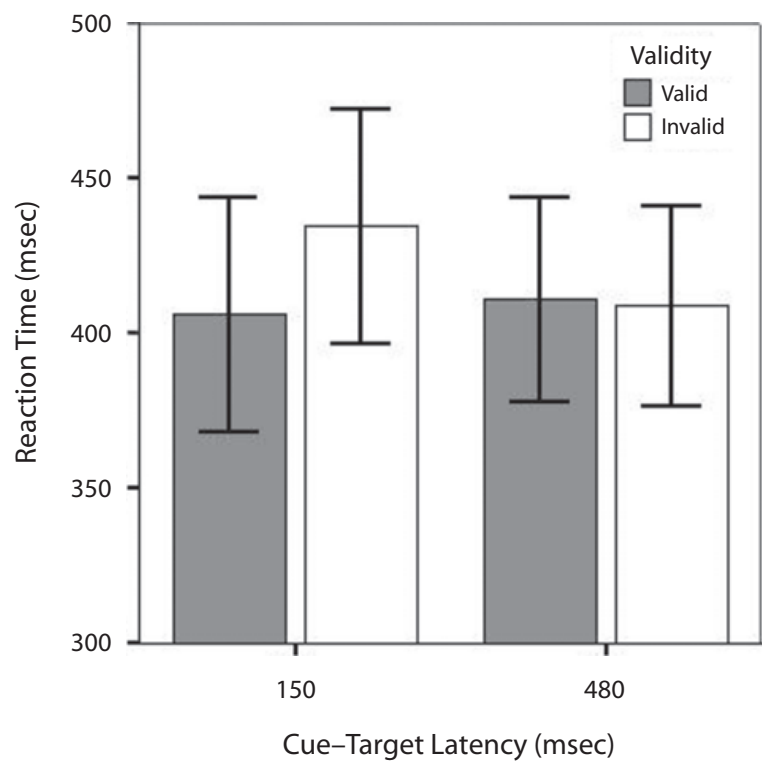

Figure 2. Mean reaction times for correct responses during the Posner cuing task (Experiment 1). Gray bars represent trials on which the cue and the target appeared at the same location (valid trials), and white bars represent trials on which the cue and the target appeared at different locations (invalid trials). Error bars show $\pm 1 S E M$. There was a significant facilitation at the cued location for the 150-msec stimulus onset asynchrony (SOA), but not for the 480-msec SOA. 


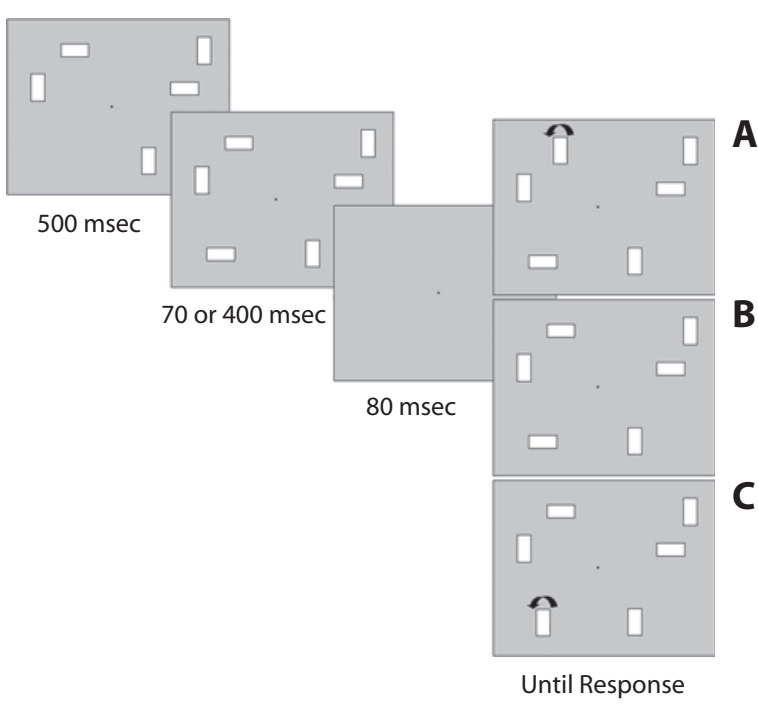

Figure 3. Procedure during the change blindness paradigm (Experiment 2). The procedure was similar to that in Experiment 1 (see Figure 1). It differed only with respect to the final display. On the final display, no probe was presented, but one of the rectangles changed its orientation on some of the trials. The participants had to indicate whether a change had occurred or not. Panel A shows trials on which change occurred at an uncued location (invalid trials), panel B shows no-change trials, and panel $\mathrm{C}$ shows trials on which the change occurred at the cued location (valid trials). The semicircular arrows illustrate the nature of the change and its location.

cesses. In contrast to the RT data, the peripheral cue had no effect on the participants' ability to correctly detect the onset of the probe stimulus. This appears to have been due to a ceiling effect, since the participants correctly detected the probe on $91.25 \%$ of the valid trials and $93.8 \%$ of the invalid trials.

These data have two important implications. First, they confirm that the sudden onset reflexively attracts attention. Second, they confirm that the effects of this reflexive attentional shift do not persist until $480 \mathrm{msec}$ after the cue onset. Experiment 1 has thus established the effect and time course of reflexive shifts in covert attention. If the reflexive attentional shift produced by the sudden onset also attenuates change blindness, we would predict that change blindness would be reduced at the cued location, but only at the first latency (i.e., $150 \mathrm{msec}$ ), and not at the longer latency (i.e., $480 \mathrm{msec}$ ).

\section{EXPERIMENT 2}

A second experiment was conducted to investigate the extent to which exogenously manipulating the locus of attention would affect participants' susceptibility to change blindness. As with the previous experiment, a sudden onset was used to reflexively attract attention to one item in the array. If it is necessary to attend to an object in order to become aware of changes to that object, we should observe facilitated change detection at the cued location, but only for a short period of time. At longer latencies, the withdrawal of attention from the cued location should correspond with decreased ability to detect changes at that location.

\section{Method}

Participants and Apparatus. Experiment 2 employed the same participants and apparatus as those in Experiment 1.

Stimuli. Experiment 2 employed the same stimuli as those in Experiment 1.

Procedure. Participants were presented with a display consisting of a gray background with a black fixation point at the center. After 1,500 msec, five white rectangles appeared, followed $500 \mathrm{msec}$ later by a sixth rectangle. After a variable latency (70 or $400 \mathrm{msec}$ ), the display was occluded for $80 \mathrm{msec}$ by a gray mask, after which the display of six rectangles reappeared. On $66 \%$ of the trials, a $90^{\circ}$ rotation of one of the rectangles occurred (change trials). On the remaining $34 \%$ of the trials, no rotation occurred (nochange trials). On $16 \%$ of the change trials, the rotation occurred at the location of the sudden onset (valid trials). On the remaining $84 \%$ of the change trials, the rotation occurred at one of the uncued locations (invalid trials). The participants were told to report whether or not they saw a change in the display by pressing the appropriate response key as quickly as possible. Each participant completed 10 practice trials and 360 experimental trials (i.e., in the change condition, there were 20 valid and 100 invalid trials at each SOA). Each participant also completed a block of 90 baseline trials on which all six rectangles appeared simultaneously (i.e., there was no sudden-onset precue). In this condition, changes occurred on $66 \%$ of the trials. Half the participants received the experimental trials first; the other half received baseline trials first. Figure 3 illustrates the course of events on a typical trial.

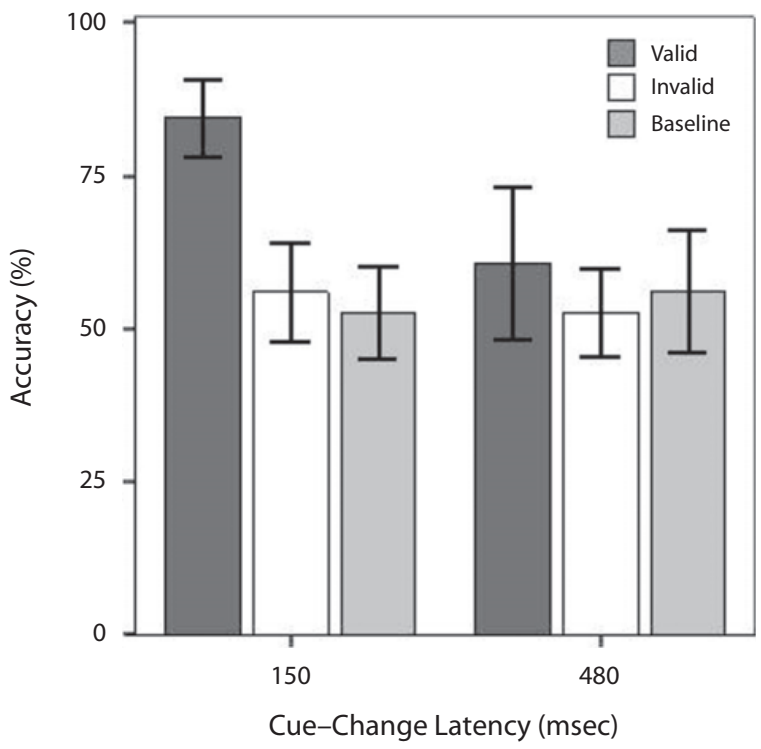

Figure 4. Accuracy of change detection in Experiment 2. Dark gray bars represent trials on which the cue and the target appeared at the same location (valid trials), white bars represent trials on which the cue and the target appeared at different locations (invalid trials), and light gray bars represent trials on which there was no sudden-onset precue (baseline trials). Error bars show $\pm 1 S E M$. Change detection was significantly facilitated at the cued location during the 150-msec stimulus onset asynchrony (SOA), but not during the 480-msec SOA. 


\section{Results}

Data analysis. Responses were filtered to remove any trials on which the participants made a saccade before the onset of the mask and responses that were more than 2.5 standard deviations longer than an individual's mean RT. Any eye movement of an amplitude of $>2^{\circ}$ was considered to be a saccade. These procedures resulted in the rejection of $3.1 \%$ of the trials $(2.7 \%$ RT errors, $0.4 \%$ saccade errors $)$.

Detection accuracy. The participants' mean accuracy scores on the change trials were subjected to a 2 (SOA: 150 or $480 \mathrm{msec}) \times 3$ (validity: valid, invalid, or no cue) repeated measures ANOVA. This analysis revealed a significant main effect of validity $[F(1,2)=5.825, p<.05]$ and a significant SOA $\times$ validity interaction $[F(1,2)=9.608, p<.05]$. An inspection of Figure 4 suggests that the interaction was driven by facilitation of discrimination accuracy at the cued location during the 150-msec SOA that was not present during the 480-msec SOA. Analysis of simple main effects (paired sample $t$ tests) confirms these impressions. Detection accuracy was significantly better on valid trials than on baseline trials $[t(1,5)=4.985, p<.05]$ and invalid trials $[t(1,5)=$ $5.584, p<.05]$. Neither of these effects were observed for the 480 -msec SOA [valid vs. invalid, $t(5)=0.860, p=.429$; valid vs. baseline, $t(5)=0.502, p=.637$ ].

Reaction time. The participants'mean RTs on the change trials were subjected to a 2 (SOA: 150 or $480 \mathrm{msec}$ ) $\times$ 3 (validity: valid, invalid, or no cue) repeated measures ANOVA. This analysis revealed no significant main effects and no interactions. However, planned contrasts (paired

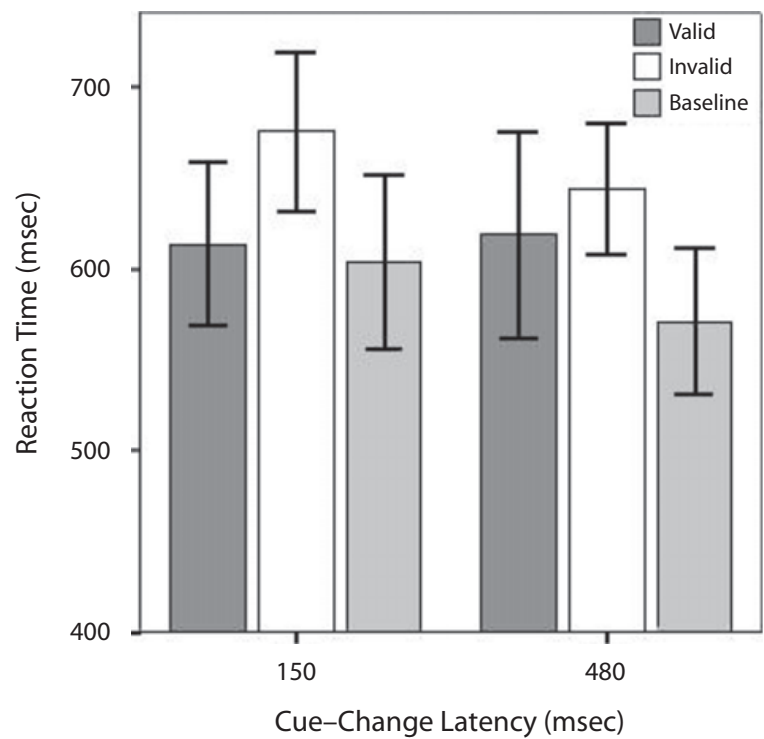

Figure 5. Mean reaction times for correct responses during the change detection task (Experiment 2). Dark gray bars represent trials on which the cue and the target appeared at the same location (valid trials), white bars represent trials on which the cue and the target appeared at different locations (invalid trials), and light gray bars represent trials that were not preceded by a cue (baseline trials). Error bars show $\pm 1 S E M$. There was a significant cuing effect at the 150-msec stimulus onset asynchrony (SOA), but not at the $480-\mathrm{msec} S O A$. sample $t$ tests) revealed that RTs were significantly shorter for the valid than for the invalid trials at the 150-msec SOA [614 vs. $675 \mathrm{msec} ; t(5)=3.286, p<.05]$, but not at the 480 -msec SOA [618 vs. 644 msec; $t(5)=0.699, p=.52$ ]. Intriguingly, RTs during valid trials were not significantly shorter than those obtained during baseline trials [613 vs. $603 \mathrm{msec} ; t(5)=0.22, p=.83$ ]. Figure 5 illustrates these RT data.

\section{Discussion}

The sudden-onset cue had a striking effect on change detection. When the change occurred within $150 \mathrm{msec}$ of the cue, detection was greatly facilitated at the cued location. However, when the change occurred $480 \mathrm{msec}$ after cue onset, there was no significant difference in detection accuracy between the cued and the uncued locations. Indeed, for the 480-msec SOA, detection was not significantly different from that in the baseline no-cue condition. Valid cues also produced significantly shorter RTs for the 150-msec SOA, but not for the 480-msec SOA. However, this effect is difficult to interpret, given the long overall RTs (around $650 \mathrm{msec}$ ) and the fact that this cuing effect appeared to be driven by a slowing of responses during invalid trials, rather than a facilitation of responses during valid trials, relative to baseline.

These results are consistent with the suggestion that attention is a necessary precondition for a change to enter into awareness. The results are unlikely to have been the consequence of a voluntary shift of attention, since the benefits occurred too early and the cue itself provided no information about the location of the change. Furthermore, the possibility that performance reflected the foveation of the changing object can be excluded, since trials on which any saccade away from fixation was made were rejected.

\section{GENERAL DISCUSSION}

The goal of the present study was to establish that covert attention, rather than proximity to fixation, is required for an observer to become aware of a change. To do this, we first established the time course of reflexive attentional shifts in response to sudden onsets in a modified version of the Posner (Posner \& Cohen, 1984) cuing paradigm. We observed cuing effects at short (150-msec) cue-target latencies that were not present at longer (480-msec) latencies. On the basis of these results, it was predicted that a peripheral cue would also modulate change detection at short, but not long, latencies. Exactly this pattern of results was observed in our change blindness experiment. These data demonstrate that covert attention can facilitate change detection and are consistent with the hypothesis that attention is a necessary precondition for visual awareness.

Intriguingly, on first inspection, our results appear to be contrary to those of Scholl (2000), who observed that sudden onsets produced a sustained facilitation of change detection despite the change's occurring $480 \mathrm{msec}$ after the onset of the new item (i.e., outside the time at which peripheral cues are known to facilitate target detection). 
In contrast, we observed that peripheral cues produced a short-lived facilitation of change detection $150 \mathrm{msec}$ after cue onset that was no longer observable $480 \mathrm{msec}$ after cue onset. However, the task utilized by Scholl differed substantially from our one-shot paradigm, in that eye movements were not constrained in his study and the change continued to be presented until it was detected. It is therefore probable that the sustained facilitation observed by Scholl was the product of either a voluntary attentional shift or an overt eye movement toward the cued location (indeed, Scholl notes this possibility in his article), rather than an effect of attentional capture.

A study by Tse, Sheinberg, and Logothetis (2003) investigated the effects of a peripheral cue on participants' ability to report the color of a sudden onset that appeared simultaneously with a secondary visual transient. They reported that participants were more accurate at detecting the color of the sudden onset when it appeared both at the cued location and opposite to the cued location. Fixations were monitored during this task, so the improved color discrimination could not be attributed to an overt eye movement to the cued location. Crucially, however, Tse et al. presented the changed object on every trial. Thus, the participants were not required to report whether or not they perceived a change in the display but, rather, to guess the color of a new object. As Tse et al. noted, their data cannot distinguish conscious change detection (which requires awareness of the change) from successful color discrimination at the cued location (which does not require awareness of there having been a change) or some other, unconscious mechanism that might improve guessing. The data in Tse et al. demonstrate that a large visual transient does not disrupt the allocation of reflexive attention, but they do not speak to the issue of whether or not an attentional shift is necessary for visual awareness.

Cole and colleagues (Cole, Kentridge, Gellatly, \& Heywood, 2003; Cole, Kentridge, \& Heywood, 2004) have previously demonstrated that sudden onsets are significantly more resistant to change blindness than are other types of change. However, it is important to note that Cole and colleagues were interested in using the change blindness paradigm to establish the special perceptual status of novel objects, rather than to establish the role of attention in visual awareness. As a consequence, there are a number of reasons why the findings by Cole et al. cannot be used to test whether attention facilitates change awareness. First, the studies did not measure eye movements and, therefore, are subject to the same problem as the Scholl (2000) study. Second, Cole and colleagues suggested that it is the special perceptual status of a novel object that makes an object onset more immune to change blindness than is a change in the appearance of an already present object. Critically, the change to be detected, in this instance, was the onset of a novel object. As a consequence, the reduced change blindness could be a consequence of a reflexive attentional shift to an object that has newly appeared or could reflect the "special" status of new object that are not subject to change blindness. The data of Cole and colleagues do not permit these two, equally likely possibilities to be dissociated. This prob- lem was avoided in our paradigm, since although we used a sudden onset to cue attention to a particular location of the visual scene, we then used a different change to probe for change awareness. This manipulation allowed us to dissociate the nature of the change from the attentionalcuing effect that a sudden onset introduced and, moreover, allowed us to study the temporal time course of such cuing effects on change detection.

It is of interest to note that although the precue caused a significant facilitation of both target detection (Posner paradigm; Experiment 1) and change detection (change blindness paradigm; Experiment 2) at the 150-msec latency, there was no evidence of an inhibition of return effect at the 480-msec latency. A number of studies have shown that task difficulty can influence the time course of facilitatory/inhibitory effects induced by a peripheral precue (e.g., Klein, 2000). The effects of a peripheral precue are typically studied in displays with only two or three potential target locations that do not change from trial to trial. In contrast, the present experiment used displays of six items whose placement and orientation were randomized across different trials. Furthermore, in order to make this task as similar as possible to the change blindness task, the display was occluded for $80 \mathrm{msec}$ prior to target onset. It may well be that these manipulations made the task sufficiently difficult to delay the onset of inhibitory processes until after the 480-msec SOA used in our task.

A second observation that warrants further comment was the high level of detection accuracy observed in the Posner cuing task (Experiment 1). The participants accurately detected the appearance of the probe stimulus on more than $90 \%$ of the trials, despite the presence of a fullfield flicker that is known to interfere with the detection of changes in visual displays and that reduced accurate change detection to $\sim 50 \%$ in Experiment 2 . One possible explanation for this difference between probe and change detection is that the probe target was a sudden onset, and sudden onsets appear to be much less susceptible to change blindness than are other forms of change, such as the orientation change used in Experiment 2 (Cole et al., 2003; Cole et al., 2004). Intriguingly, the validity of the precue did not have any effect on detection accuracy. This finding appears to be in contrast with that of Tse et al. (2003), who reported that precues did facilitate color discriminations of sudden onsets. However, the similarity between the probe and the distractor stimuli in Tse et al.'s study was much greater than that in our paradigm (a $0.69^{\circ} \times$ $0.69^{\circ}$ red or green onset among 149 similarly sized red and green placeholders, as opposed to a black asterisk in a white box). Furthermore, the participants in Tse et al. were asked to make a difficult color discrimination, whereas our participants had to perform a simple detection task. It seems plausible that differences in the relative difficulty of the discrimination tasks can account for the differing effects of the precue in the two studies.

In summary, none of the previously conducted studies provided clear evidence for a link between reflexive attention and change detection. Our study is, therefore, in our view, the first one to demonstrate this link unequivocally. Furthermore, our study also makes two additional 
contributions. First, we established that the time course of attentional cuing in the change blindness paradigm is comparable to that in a Posner paradigm. This similarity suggests that the mechanisms underlying both attentionalcuing effects are the same. If similar mechanisms mediate cuing effects in both the Posner paradigm and our change blindness paradigm, it seems reasonable to infer that the neural structures that have been demonstrated to facilitate discrimination at the cued location in a Posner paradigm are probably also critical for determining whether a change enters visual awareness or not. Second, although the Posner paradigm has proved to be one of the most popular and influential paradigms in the study of attention, the cuing effect can be small and unreliable. In contrast, the cuing effects obtained in our Experiment 2 are substantial and can be confidently demonstrated even in small samples. This paradigm therefore recommends itself as a more reliable tool for studying attention in humans.

\section{AUTHOR NOTE}

This research was supported by the Wolfson Research Institute (University of Durham). Correspondence concerning this article should be addressed to D. T. Smith, Cognitive Neuroscience Research Unit, Wolfson Research Institute, University of Durham, Queens Campus, Stockton-onTees TS17 6BH, England (e-mail: daniel.smith2@durham.ac.uk).

\section{REFERENCES}

Bridgeman, B., Hendry, D., \& STARK, L. (1975). Failure to detect displacement of visual world during saccadic eye movements. Vision Research, 15, 719-722.

Cole, G. G., Kentridge, R. W., Gellatly, A. R. H., \& Heywood, C. A. (2003). Detectability of onsets versus offsets in the change detection paradigm. Journal of Vision, 3, 22-31.

Cole, G. G., Kentridge, R. W., \& Heywood, C. A. (2004). Visual salience in the change detection paradigm: The special role of object onset. Journal of Experimental Psychology: Human Perception \& Performance, 30, 464-477.

Deubel, H., \& Schneider, W. X. (1996). Saccade target selection and object recognition: Evidence for a common attentional mechanism. Vision Research, 36, 1827-1837.
Hoffman, J. E., \& Subramaniam, B. (1995). The role of visual attention in saccadic eye movements. Perception \& Psychophysics, 57, 787-795.

Kelley, T. A., Chun, M. M., \& ChuA, K.-P. (2003). Effects of scene inversion on change detection of targets matched for visual salience. Journal of Vision, 3, 1-5.

KLEIN, R. M. (2000). Inhibition of return. Trends in Cognitive Sciences, 4, 138-147.

MAYLOR, E. A., \& Hockey, R. (1985). Inhibitory component of externally controlled covert orienting in visual space. Journal of Experimental Psychology: Human Perception \& Performance, 11, 777-787.

McConkie, G. W., \& Zola, D. (1979). Is visual information integrated across successive fixations in reading? Perception \& Psychophysics, 25, 221-224

MülleR, H. J., \& Rabbitt, P. M. A. (1989). Reflexive and voluntary orienting of visual attention: Time course of activation and resistance to interruption. Journal of Experimental Psychology: Human Perception \& Performance, 15, 315-330.

O'Regan, J. K., Deubel, H., Clark, J. J., \& Rensink, R. A. (2000). Picture changes during blinks: Looking without seeing and seeing without looking. Visual Cognition, 7, 191-211.

O’Regan, J. K., Rensink, R. A., \& Clark, J. J. (1999). Change-blindness as a result of "mudsplashes." Nature, 398, 34.

Pashler, H. (1988). Familiarity and visual change detection. Perception \& Psychophysics, 44, 369-378

Phillips, W. A. (1974). On the distinction between sensory storage and short-term visual memory. Perception \& Psychophysics, 16, 283-290.

Posner, M. I., \& Cohen, Y. (1984). Components of visual orienting. In H. Bouma \& D. G. Bouwhuis (Eds.), Attention and performance X: Control of language processes (pp. 531-556). Hillsdale, NJ: Erlbaum.

Rensink, R. A., O'Regan, J. K., \& Clark, J. J. (1997). To see or not to see: The need for attention to perceive changes in scenes. Psychological Science, 8, 368-373.

Scholl, B. J. (2000). Attenuated change blindness for exogenously attended items in a flicker paradigm. Visual Cognition, 7, 377-396.

ShePherd, M., Findlay, J. M., \& Hockey, R. J. (1986). The relationship between eye-movements and spatial attention. Quarterly Journal of Experimental Psychology, 38A, 475-491.

Simons, D. J., \& Rensink, R. A. (2005). Change blindness: Past, present, and future. Trends in Cognitive Sciences, 9, 16-20.

Tse, P. U., Sheinberg, D. L., \& Logothetis, N. K. (2003). Attentional enhancement opposite a peripheral flash revealed using change blindness. Psychological Science, 14, 91-99.

(Manuscript received April 13, 2007; revision accepted for publication October 18, 2007.) 